\title{
DIABETES
}

\section{Hepatic lipogenesis independent of insulin in type 2 diabetes mellitus-a paradox clarified}

A new study provides clues to reconcile the apparently paradoxical observation that the livers of patients with type 2 diabetes mellitus (T2DM) continue to actively synthesize triglycerides despite being resistant to the effects of insulin.

In $\mathrm{T} 2 \mathrm{DM}$, the continued production of triglycerides by the liver and the concomitant failure to suppress glucose production lead to hyperglycaemia, hyperlipidaemia and hepatic steatosis. Triglycerides are synthesized in the liver via esterification of fatty acids, which have different origins. Hepatic fatty acids can result from local synthesis from acetyl CoA (de novo lipogenesis), but they can also result from direct uptake from plasma. Prior findings indicated that the plasma is the major source of triglyceride synthesis in patients with $\mathrm{T} 2 \mathrm{DM}(>60 \%)$, but the role of insulin in fatty acid esterification, if any, was not clear.

Shulman and colleagues investigated whether the synthesis of triglycerides from pre-formed fatty acids is independent of insulin. The researchers developed a novel liquid chromatography-tandem mass spectrometry method to measure the rates of fatty acid esterification in vivo in several rat models: normal rats, insulin-resistant rats fed a high-fat diet and rats treated with an antisense oligonucleotide that blocks expression of the insulin receptor in the liver. Fatty acids and insulin infusions were performed to assess how variations in the levels of these molecules in plasma affect the rates of hepatic fatty acid esterification.

"In contrast to hepatic de novo lipogenesis, we found that the rates at which fatty acids were transformed into triglycerides primarily depended on fatty acid delivery to the liver and occurred independently of hepatic insulin action," says Shulman. "These results explain why insulin therapy, rather than exacerbating dyslipidaemia and nonalcoholic fatty liver disease (NAFLD) in patients with T2DM, actually helps to ameliorate

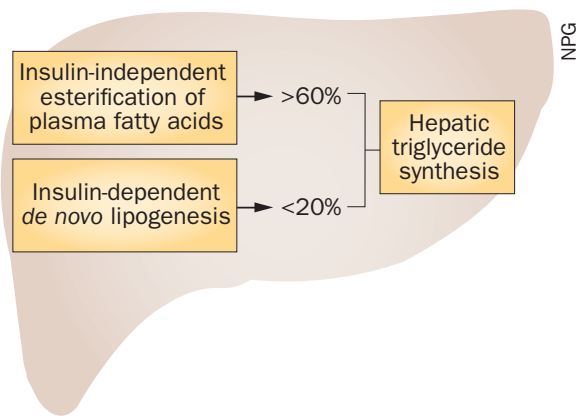

diabetic dyslipidaemia and NAFLD in these patients. Furthermore, this work emphasizes that therapies targeting improved adipose tissue function and suppression of lipolysis will be valuable for the treatment of NAFLD and hepatic insulin resistance."

Shulman and colleagues plan to use ${ }^{13} \mathrm{C}$ magnetic resonance spectroscopy to see how their findings about the regulation of fatty acid esterification in rats translate to humans.

Joana Osório

Original article Vatner, D. F. et al. Insulin-independent regulation of hepatic triglyceride synthesis by fatty acids. Proc. Natl Acad. Sci. USA doi:10.1073/pnas.1423952112 